\title{
Complementarity for Generalized Observables
}

\author{
Alfredo Luis* \\ Departamento de Óptica, Facultad de Ciencias Físicas, Universidad Complutense, 28040 Madrid, Spain
} (Received 16 September 2001; published 22 May 2002)

\begin{abstract}
We examine basic properties of complementarity by using the most general description of quantum observables as positive-operator measures. We show that, in general, two observables can be complementary or not depending on the measure of fluctuations adopted and that complementarity is not a symmetric relation. This occurs because the states that determine the measured statistics do not necessarily coincide with the minimum uncertainty states for the same observable. We also show that there are observables without a complementary observable and that complementarity is not preserved by the Neumark extensions.
\end{abstract}

DOI: $10.1103 /$ PhysRevLett.88.230401

Complementarity is at the conceptual heart of the quantum theory since it is a direct consequence of the superposition principle. This concept has no classical analog and implies that quantum systems possess properties that are mutually exclusive: The observation of one of them precludes the observation of the other. In other words, precise knowledge of one of them implies that all possible outcomes of measuring the other one are equally probable [1]. Complementarity is a very intuitive and appealing concept that has attracted a lot of attention from the beginning of the quantum theory. However, only recently this concept has been thoroughly scrutinized and formalized. This examination includes formal definitions, quantitative assessment, experimental observation, and investigation of its physical origin $[1,2]$. It can be appreciated in Refs. [1,2] that this remarkable effort has been mainly developed in the past decade.

It must be noted that most approaches to complementarity focus on observables represented by Hermitian operators. However, in recent times it has been put forward that this standard quantum-mechanical correspondence is actually too narrow and does not encompass important physical quantities such as elapsed time or the harmonic-oscillator phase, for example. The most general representation of quantum observables is provided by positive-operatorvalued measures (POVMs), also known as generalized observables [3]. In this general context, the observables represented by Hermitian operators are particular examples arising when the POVM is made of mutually orthogonal projectors. These are referred to as projectionvalued measures (PVMs).

In this work, we examine which are the basic and universally valid properties of complementarity when considering the most general class of quantum observables. We show that basic properties that are valid for Hermitian operators no longer apply when considering generalized observables. More specifically, we show that two observables can be complementary or not depending on the measure of fluctuations adopted, that complementarity is not a symmetric relation, and also that there are observables without a complementary. Finally, we discuss whether the
PACS numbers: 03.65.Ta, 42.50.Dv

possible noisy character of generalized observables may explain these results by using Neumark extensions [3].

To begin with, we think it is convenient to recall some basic properties usually linked to the standard understanding of complementarity that we will show later are valid only when representing observables by Hermitian operators (PVMs). For the sake of simplicity and without loss of generality, we focus on a system describable by a finitedimensional Hilbert space. To be more specific, we consider that this space describes an angular momentum $j$. We denote by $|m\rangle$, with $m=-j,-j+1, \ldots, j$, the orthonormal basis defined by the eigenvectors of an angularmomentum component. As a valid representative of an arbitrary PVM, we consider $M(m)=|m\rangle\langle m|$, and we will refer globally to this observable as $M$.

The first property we recall is that there is always a PVM complementary to $M$. For example, this is the case of the PVM defined as $K(k)=|k\rangle\langle k|$, where $|k\rangle$ is the orthonormal basis,

$$
|k\rangle=\frac{1}{\sqrt{2 j+1}} \sum_{m=-j}^{j} e^{i[2 \pi /(2 j+1)] k m}|m\rangle,
$$

and $k=1,2, \ldots, 2 j+1$ are integers. We will refer to this PVM as $K$. The states (1) are the angle-phase states for finite-dimensional systems [4,5]. Since $|\langle k \mid m\rangle|$ is constant, we have that for the states conveying precise knowledge of $M$ (the states $|m\rangle$ ) all possible outcomes of measuring $K$ are equally probable, so that $K$ is complementary to $M$. Therefore, every PVM has at least one complementary PVM.

It is worth pointing out that there is no need to resort to any measure of uncertainty in order to assert that $|m\rangle$ are the states conveying precise knowledge of $M$. Therefore, for PVMs, complementarity is a property that does not depend on any measure of fluctuations. The last property we recall is that the complementarity between $K$ and $M$ is actually a symmetric relation since $|k\rangle$ and $|m\rangle$ can naturally interchange their roles.

Next we show that these three properties (existence, symmetry, and irrelevance of uncertainty measures) no longer apply when considering arbitrary observables. The 
POVMs provide the most general correspondence between quantum states, represented by a density matrix $\rho$, and the statistics $P(x)$ of a measuring arrangement $P(x)=$ $\operatorname{tr}[\rho \Delta(x)]$, where $\Delta(x)$ is a family of linear operators. The natural properties of probabilities (real, positive, and normalized) are the only requirements imposed on $\Delta(x)$, leading to the conditions $\Delta^{\dagger}(x)=\Delta(x), \Delta(x) \geq 0$, and $\int d x \Delta(x)=I$, where $I$ is the identity. This general correspondence encompasses the Hermitian operators as particular cases (PVMs).

Let us focus on the POVM, which we shall denote as $\Phi$, defined as $\Phi(\phi)=|\phi\rangle\langle\phi|$, where $|\phi\rangle$ are the nonorthogonal and unnormalized vectors,

$$
|\phi\rangle=\frac{1}{\sqrt{2 \pi}} \sum_{m=-j}^{j} e^{i \phi m}|m\rangle,
$$

and $\phi$ can take any value in a $2 \pi$ interval. We stress that this is not a PVM (it does not admit a Hermitian operator) since the vectors $|\phi\rangle$ are not orthogonal $\left\langle\phi^{\prime} \mid \phi\right\rangle \not \subset \delta\left(\phi^{\prime}-\right.$ $\phi)$. Because of this lack of orthogonality, there is no state having a definite nonfluctuating value for $\Phi$, and all states have a probability distribution $P(\phi)=\operatorname{tr}[\Phi(\phi) \rho]$ having a nonzero width $[5,6]$. In our context, this implies that there is no trivial answer to the question of which of the states are conveying precise knowledge of $\Phi$. On what follows we will interpret precise knowledge as being equivalent to minimum uncertainty.

In order to find observables complementary to $\Phi$, we have to find the states with minimum uncertainty for $\Phi$. This requires that one specify a measure of fluctuations. This can be done in many different ways, and we can expect that different measures will lead to different complementary observables [7]. We demonstrate this point by considering two particular measures of fluctuations: reciprocal peak and dispersion.

According to the reciprocal peak criterion, the states with minimum uncertainty are those with the highest absolute maximum of $P(\phi)[8,9]$. It is easy to see that these states are the phase states (2). Since $|\langle m \mid \phi\rangle|$ is constant, we conclude that $M$ is complementary to $\Phi$.

On the other hand, instead of reciprocal peak we can consider the dispersion $\delta \phi$ as a measure of phase uncertainty [9-11]:

$$
(\delta \phi)^{2}=1-\left|\int_{2 \pi} d \phi e^{i \phi} P(\phi)\right|^{2} .
$$

It can be seen that the states with minimum dispersion are [12]

$$
|\tilde{\phi}\rangle=\frac{1}{\sqrt{j+1}} \sum_{m=-j}^{j} \sin \left[\frac{(j+m+1)}{2 j+2} \pi\right] e^{i m \phi}|m\rangle,
$$

where $\phi$ can take any value. For $j>1 / 2$ we have that $|\langle m \mid \tilde{\phi}\rangle|$ depends on $m$ and thus $M$ is no longer complementary to $\Phi$. The conclusion is that in the most general case two observables are complementary or not depending on the measure of fluctuations adopted.

This very same example can serve to demonstrate that complementarity is not a symmetric relation. We have just shown that the states $|m\rangle$ with well-defined $M$ have a uniform probability distribution for $\Phi$ and, hence, $\Phi$ is complementary to $M$. However, the states $|\tilde{\phi}\rangle$ having well-defined $\Phi$ (according to the dispersion criterion) have not a uniform probability distribution for $M$, and thus $M$ is not complementary to $\Phi$.

The origin of this lack of symmetry is that the states with minimum fluctuations $|\tilde{\phi}\rangle$ can be different from the states $|\phi\rangle$ that determine the statistics via projection. They coincide if the observable is represented by a PVM, but they can be different when the observable is represented by a POVM. In other words, the states that represent a physical quantity when it is measured are different from the states that carry precise information about the same quantity. This is a remarkable and an almost paradoxical result. Further consequences are discussed below.

Finally, we demonstrate that there are generalized observables without complementarity. Let us consider the $\operatorname{POVM} q(\Omega)=[(2 j+1) /(4 \pi)]|\Omega\rangle\langle\Omega|$ defined in terms of the SU(2) coherent states $|\Omega\rangle$ [13]. There are measures of fluctuations, such as reciprocal peak, that lead to the $\mathrm{SU}(2)$ coherent states $|\Omega\rangle$ as the minimum uncertainty states. Following such criteria, any potential POVM $p$ complementary to $q$ should satisfy that $\langle\Omega|p(\ell)| \Omega\rangle$ does not depend on $\ell$, where $\ell$ is the index labeling the elements of $p$. Because of the normalization of probabilities, $\sum_{\ell}\langle\Omega|p(\ell)| \Omega\rangle=1$, we have that $\langle\Omega|p(\ell)| \Omega\rangle$ does not depend on $\Omega$. This means that the $\operatorname{SU}(2) Q$ function of the operators $p(\ell)$ is constant and, hence, $p(\ell)$ is proportional to the identity. Therefore, since the only observable complementary to $q$ is trivial, we can say that $q$ has no complementary observables. It is worth noting that the POVM $q(\Omega)$ is an informationally complete measurement since the corresponding statistics allows one to determine the whole statistics of any other observable. In our context this completeness is reflected by the lack of complementary observables.

We can recall that every POVM can be represented by a PVM acting on an enlarged Hilbert space (this is the Neumark extension, also spelled Naimark in the literature). In principle, we may expect that this correspondence would be useful to elucidate quantum properties of POVMs (complementarity, for example) by using the more standard representation of quantum observables by Hermitian operators. However, the preceding results suggest that the Neumark extensions do not preserve complementarity. In what follows, we demonstrate this fact by means of two particular examples.

To this end, we consider Neumark extensions where the enlarged space is made of two independent harmonic oscillators with annihilation operators $a_{1}$ and $a_{2}$ acting on the corresponding Hilbert spaces $\mathcal{H}_{1}$ and $\mathcal{H}_{2}$, respectively. 
For the sake of definiteness, we assume that they represent two quantum electromagnetic field modes. The reduced system where the POVMs of interest are defined is the mode $a_{1}$, while the auxiliary mode $a_{2}$ is in a fixed state, the vacuum, for example, $|0\rangle_{2} \in \mathcal{H}_{2}$. Since the state in mode $a_{2}$ is fixed and known in advance, any measurement performed on the whole system $\mathcal{H}_{1} \otimes \mathcal{H}_{2}$ can be regarded as providing information about the state of the signal mode $a_{1}$. In this way a PVM in $\mathcal{H}_{1} \otimes \mathcal{H}_{2}$ becomes a POVM in $\mathcal{H}_{1}$.

After these definitions, we demonstrate that two complementary PVMs in $\mathcal{H}_{1} \otimes \mathcal{H}_{2}$ can give two observables in $\mathcal{H}_{1}$ that are not complementary. Vice versa, we will also show that two PVMs in $\mathcal{H}_{1} \otimes \mathcal{H}_{2}$ that are not complementary can lead to two observables that are complementary in $\mathcal{H}_{1}$.

In order to demonstrate the first statement, let us consider PVMs in $\mathcal{H}_{1} \otimes \mathcal{H}_{2}$ compatible with the total photon number so that they are given by projection on some orthogonal basis of the form

$$
|n, \alpha\rangle=\sum_{k=0}^{n} c_{n, k}(\alpha)|n-k\rangle_{1}|k\rangle_{2},
$$

where $n$ represents the total photon number, $\alpha$ is the rest of the parameters required to suitably label the basis, and $|n\rangle_{1,2}$ represent photon number states in the corresponding mode. Taking into account that mode $a_{2}$ is always in the vacuum state, the PVMs $|n, \alpha\rangle\langle n, \alpha|$ in $\mathcal{H}_{1} \otimes \mathcal{H}_{2}$

$$
|k, \varphi\rangle=\frac{1}{\sqrt{2 \pi}}\left[|k\rangle_{1}|k\rangle_{2}+\sum_{\ell=1}^{\infty}\left(e^{i \ell \varphi} \mid \ell\right.\right.
$$

and are the eigenstates of the so-called relative-phase operator expressed in the photon number basis $[5,14]$. These are Neumark extensions of the desired observables since ${ }_{2}\langle 0 \mid n, m\rangle \propto|n\rangle_{1}$, and ${ }_{2}\langle 0 \mid k=0, \varphi\rangle \propto|\varphi\rangle_{1}$.

It is easy to show that these Neumark extensions are not complementary. For example, we have

$$
\langle k=0, \varphi \mid n, m=n\rangle=\frac{\cos (n \varphi)}{\sqrt{2^{n-1} \pi}}\left(1-\frac{1}{2} \delta_{n, 0}\right),
$$

which is not a constant.

Finally, we discuss the results presented in this work and some of their implications. For example, it has been recently disputed whether complementarity is logically independent of uncertainty relations $[1,2]$. The results of this paper deepen this issue since they imply that even the very definition of complementarity depends on the measure of fluctuations adopted. Furthermore, this must have consequences concerning the quantitative assessment of complementarity. We can no longer say simply that wave behavior (represented here by the phase) and particle behavior (represented here by the number) are mutually exclusive. The use of epithets such as mutually exclusive or duality must be revised in light of the results of this work. become the following POVMs in $\mathcal{H}_{1}$ :

$$
\Delta(n, \alpha)=\operatorname{tr}_{2}\left(|0\rangle_{22}\langle 0|| n, \alpha\rangle\langle n, \alpha|\right) \propto|n\rangle_{11}\langle n|,
$$

which is independent of $\alpha$. This means that every measurement in $\mathcal{H}_{1} \otimes \mathcal{H}_{2}$ compatible with the total photon number gives exactly the same reduced observable in $\mathcal{H}_{1}$. In particular, this applies to two complementary observables, such as the number difference $\left[c_{n, k}(\alpha)=\delta_{k, \alpha}\right]$ and the phase difference $\left[c_{n, k}(\alpha)=e^{i \alpha k} / \sqrt{n+1}\right]$.

Next, we show that two PVMs in $\mathcal{H}_{1} \otimes \mathcal{H}_{2}$ that are not complementary can lead to two observables that are complementary in $\mathcal{H}_{1}$. The complementary observables in $\mathcal{H}_{1}$ are the photon number and the phase variable defined in terms of the Susskind-Glogower states $[4,8,10]$ :

$$
|\varphi\rangle_{1}=\frac{1}{\sqrt{2 \pi}} \sum_{n=0}^{\infty} e^{i n \varphi}|n\rangle_{1},
$$

where $\varphi$ can take any value in a $2 \pi$ interval. The phase observable $|\varphi\rangle\langle\varphi|$ is a POVM since the Susskind-Glogower states are not orthogonal. Photon number and phase are mutually complementary according to the reciprocal peak criterion since in such a case the minimum phase-uncertainty states are the Susskind-Glogower states (7) and $\left.\right|_{1}\langle\varphi \mid n\rangle_{1} \mid$ is constant. As their Neumark extensions, we consider the PVMs defined by the states $|n, m\rangle$ and $|k, \varphi\rangle$. The states $|n, m\rangle$ are the simultaneous eigenstates of the total photon number (with eigenvalue $n$ ) and the operator $a_{1}^{\dagger} a_{2}+a_{2}^{\dagger} a_{1}$ (with eigenvalues $m=$ $-n,-n+2, \ldots, n)$. The states $|k, \varphi\rangle$ are defined as

$\left.\left.+k\rangle_{1}|k\rangle_{2}+e^{-i \ell \varphi}|k\rangle_{1}|\ell+k\rangle_{2}\right)\right]$

Such statements must be accompanied by an explicit and definite choice of a measure of fluctuations or, otherwise, they are void of meaning. On the other hand, the lack of symmetry demonstrated above implies that the two components of a pair of dual observables actually play dissimilar roles.

It must be stressed that the results of this paper do not occur solely for academic or exotic observables. On the contrary, we have demonstrated these results by using very common observables such as number and phase, or angular momentum and angle.

The POVMs are sometimes characterized as having extra randomness in comparison to PVMs. It may be asked whether the results presented in this work are simple consequences of such extra randomness. The additional degrees of freedom that cause extra fluctuations are formally described by enlarging the Hilbert space (Neumark extensions). However, we have just demonstrated above that the Neumark extensions do not preserve complementarity so that they cannot explain the complementarity properties of generalized observables. Therefore, the results of this work cannot be accounted for easily in terms of extra randomness. In this context, it is worth pointing out that there 
are POVMs that are informationally complete, such as the one defined by the $\mathrm{SU}(2)$ coherent states, which do not fit with such a noisy picture. It seems that it would be difficult to remove the alleged randomness without degrading the information content conveyed by such measurements.

In the above analysis, we have focused on the idea of complementarity as a property related to the intrinsic statistical uncertainty of quantum observables. A different approach focuses on the idea of complementarity as the result of the disturbance caused by quantum measurement. Next, we briefly examine this aspect showing that the standard approach to state reduction does not imply any relevant difference between PVMs and POVMs concerning the enforcement of complementarity.

To this end, we may consider that the POVM defined by the states (2) is measured on some initial state $|\psi\rangle$. [Note that we cannot consider a measurement based on the states (4) since they do not provide a resolution of the identity.] The most general reduced state (assumed pure for simplicity) conditioned to the outcome $\phi$ is given by the unnormalized vector $|\psi(\phi)\rangle=|\varphi(\phi)\rangle\langle\phi \mid \psi\rangle$, where $|\varphi(\phi)\rangle$ are arbitrary normalized states. It is worth noting that the measured observable does not determine the reduced states $|\varphi(\phi)\rangle[15]$. The key point in our context is that this is equally valid for PVMs and POVMs. In particular, the reduced states can be different from the states that determine the statistics and also different from the states carrying precise information about the measured observable. Moreover, since the formulas describing state reduction are formally the same for PVMs and POVMs, we can refer to previous literature concerning the evaluation of the system disturbance caused by measurement [16].

*Electronic address: alluis@ fis.ucm.es

[1] M. O. Scully, B.-G. Englert, and H. Walther, Nature (London) 351, 111 (1991).

[2] X. Y. Zou, L. J. Wang, and L. Mandel, Phys. Rev. Lett. 67, 318 (1991); S. M. Tan and D. F. Walls, Phys. Rev. A 47, 4663 (1993); P. Storey, S. Tan, M. Collett, and D. Walls, Nature (London) 367, 626 (1994); B.-G. Englert, M. O. Scully, and H. Walther, ibid. 375, 367 (1995); E. P. Storey, S. M. Tan, M. J. Collett, and D.F. Walls, ibid. 375, 368 (1995); H. Wiseman and F. Harrison, ibid. 377, 584 (1995); B.-G. Englert, Phys. Rev. Lett. 77,
2154 (1996); L. S. Schulman, Phys. Lett. A 211, 75 (1996); H. M. Wiseman, F.E. Harrison, M. J. Collett, S. M. Tan, D. F. Walls, and R. B. Killip, Phys. Rev. A 56, 55 (1997); P. Knight, Nature (London) 395, 12 (1998); E. Buks, R. Schuster, M. Heiblum, D. Mahalu, and V. Umansky, ibid. 391, 871 (1998); S. Dürr, T. Nonn, and G. Rempe, ibid. 395, 33 (1998); Phys. Rev. Lett. 81, 5705 (1998); G. Björk, J. Söderholm, A. Trifonov, T. Tsegaye, and A. Karlsson, Phys. Rev. A 60, 1874 (1999); O. Steuernagel, quant-ph/9908011; A. Luis and L. L. Sánchez-Soto, Phys. Rev. Lett. 81, 4031 (1998); J. Opt. B 1, 668 (1999); S. Dürr and G. Rempe, Am. J. Phys. 68, 1021 (2000); P. Busch, P. Lahti, J.-P. Pellonpää, and K. Ylinen, J. Phys. A 34, 5923 (2001); P. Bertet, S. Osnaghi, A. Rauschenbeutel, G. Nogues, A. Auffeves, M. Brune, J. M. Raimond, and S. Haroche, Nature (London) 411, 166 (2001); M. Mei and M. Weitz, Phys. Rev. Lett. 86, 559 (2001); A. Luis, Phys. Rev. A 64, 012103 (2001); J. Phys. A 34, 8597 (2001); S. Dürr, Phys. Rev. A 64, 042113 (2001).

[3] C. W. Helstrom, Quantum Detection and Estimation Theory (Academic, New York, 1976); A. Peres, Found. Phys. 20, 1441 (1990); Quantum Theory: Concepts and Methods (Kluwer Academic, Dordrecht, 1993).

[4] T. S. Santhanam, Phys. Lett. 56A, 345 (1976); S. M. Barnett and D. T. Pegg, J. Mod. Opt. 36, 7 (1989); D. Ellinas, J. Math. Phys. (N.Y.) 32, 135 (1991).

[5] A. Luis and L. L. Sánchez-Soto, Progress in Optics, edited by E. Wolf (Elsevier, Amsterdam, 2000), Vol. 41, p. 421.

[6] M. Grabowski, Int. J. Theor. Phys. 28, 1215 (1989); Rep. Math. Phys. 29, 377 (1991).

[7] I. Bialynicki-Birula, M. Freyberger, and W. Schleich, Phys. Scr. T48, 113 (1993); M. J. W. Hall, J. Mod. Opt. 40, 809 (1993).

[8] J. H. Shapiro and S. R. Shepard, Phys. Rev. A 43, 3795 (1991).

[9] Z. Hradil, Phys. Rev. A 46, R2217 (1992).

[10] V. Peřinová, A. Lukš, and J. Peřina, Phase in Optics (World Scientific, Singapore, 1998).

[11] J. M. Lévy-Leblond, Ann. Phys. (N.Y.) 101, 319 (1976); T. Opatrný, J. Phys. A 27, 7201 (1994).

[12] A. Luis and J. Peřina, Phys. Rev. A 54, 4564 (1996).

[13] F. T. Arecchi, E. Courtens, R. Gilmore, and H. Thomas, Phys. Rev. A 6, 2211 (1972).

[14] M. Ban, Phys. Lett. A 152, 223 (1991); Opt. Commun. 94, 231 (1992); Phys. Lett. A 176, 47 (1993).

[15] J. L. Park, Found. Phys. 1, 23 (1970); K. Kraus, Ann. Phys. (N.Y.) 64, 311 (1971); C. M. Caves and G. J. Milburn, Phys. Rev. A 36, 5543 (1987); B. d'Espagnat, ibid. 38, 5450 (1988); M. Ozawa, Phys. Lett. A 282, 336 (2001).

[16] K. Banaszek, Phys. Rev. Lett. 86, 1366 (2001). 